\title{
SePARATION OF NATURAL From LABORATORY-GROWN DIAMOND USING TIME-GATED LUMINESCENCE IMAGING
}

\author{
Colin D. McGuinness, Amber M. Wassell, Peter M.P. Lanigan, and Stephen A. Lynch
}

A technique that expands on the surface luminescence imaging used in the DiamondView instrument has been developed at De Beers Group Technology, Maidenhead, UK. This provides an additional level of imaging information by way of separating prompt and delayed surface luminescence. The technique has the added benefit of quickly and easily distinguishing colorless or near-colorless natural diamond from laboratory-grown diamond. It can be applied when the identification of natural diamond is required in the study of single stones, multiples in batches, set jewelry, or in a fully automated process. The prompt and delayed luminescence characteristics of natural diamond are compared with a range of chemical vapor deposition (CVD) and high-pressure, high-temperature (HPHT) synthetic diamonds. Of significant interest are some of the less common CVD synthetic samples that have been observed in recent years. This article will summarize the luminescence observed in different diamond types, discuss its spectral characteristics, and serve as a useful reference when interpreting such luminescence images.

T he task of identifying a diamond will typically fall to a diamond appraiser, grader, or gemologist while preparing a grading certificate or appraisal. Whether a diamond is natural or lab-grown is a key factor in its market value and is of paramount importance to the gemologist. Many characteristics can be used to distinguish between natural and synthetic diamond, but the inherent variability in the properties of natural and synthetic diamond can make such a task difficult.

A useful and proven characteristic is the emission of luminescence when a diamond is excited by a source of ultraviolet energy. Typically, a gemologist would utilize an ultraviolet lamp with excitation wavelengths of $365 \mathrm{~nm}$ (long-wave ultraviolet, LWUV) or $254 \mathrm{~nm}$ (short-wave ultraviolet, SWUV), which correspond to the emission lines of a low-pressure mercury-vapor lamp. In this application, "fluorescence" would be observed during ultraviolet excitation, while "phosphorescence" may be observed when the excitation is removed.

The De Beers DiamondView instrument (Spear and Welbourn, 1994; Welbourn et al., 1996) was de-

See end of article for About the Authors and Acknowledgments.

Gems \& Gemology, Vol. 56, No. 2, pp. 220-229,

http://dx.doi.org/10.5741/GEMS.56.2.220

(C) 2020 Gemological Institute of America signed to authenticate natural diamonds and synthetic diamonds. It allows a more sophisticated observation to be made by way of a shorter wavelength excitation of $<225 \mathrm{~nm}$ corresponding to the primary absorption edge and only exciting around 1-micron depth of material, such that observed luminescence

\section{In Brief}

- Correctly identifying natural versus laboratory-grown diamond is important for its market value and maintaining consumer confidence.

- A time-gated imaging technique has been developed at De Beers Group Technology to allow easy separation of colorless/near-colorless natural and synthetic diamond.

- A blue delayed luminescence can be used as a specific marker for identification of $>99 \%$ of natural type Ila and type la diamond.

can be considered as produced at the surface. Studies have shown (Martineau et al., 2004) that such luminescence imaging techniques can provide very useful information for identifying all synthetic diamond types. That article reported the identification of all CVD synthetics under study using DiamondView 


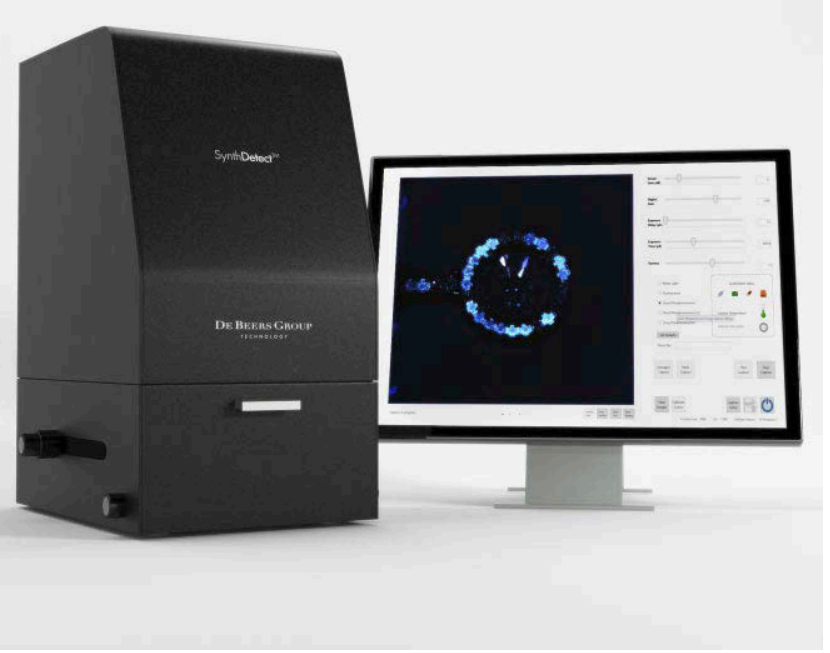

Figure 1. The De Beers SYNTHdetect, an instrument designed for screening of colorless to near-colorless diamonds of all cuts mounted in jewelry. Loose stones can also be screened with no lower size limit. The dimensions of the instrument are $31.0 \mathrm{~cm}$ wide $\times$ $34.0 \mathrm{~cm}$ deep $\times 45.8 \mathrm{~cm}$ high. A range of jewelry holders and a flat sample tray are included. Photo by Danny Bowler/De Beers Group Industry Services.

imaging, with techniques such as X-ray topography providing supporting evidence in more challenging cases. More recently, a set of color emission filters has been included with the DiamondView instrument to assist with some of the more challenging silicon-doped CVD synthetics that exhibit a degree of luminescence toward the red end of the spectrum, normally swamped by blue dislocation luminescence (Martineau, 2017).

To help negate the need for further time-consuming and expensive investigation, and to assist less experienced gemologists, the imaging technique employed by the DiamondView has been extended by way of hardware synchronization of the light source and camera (Smith et al., 2017), allowing camera exposures to be controlled relative to the lamp pulse with microsecond accuracy. As the temporal characteristics of diamond luminescence can be complex and the distinction between fluorescence and phosphorescence is not always clear, we use the term prompt luminescence to describe luminescence recorded at the same time as the lamp pulse, with delayed luminescence recorded after the lamp pulse.

There have been numerous "time-resolved" methods used to study the complex temporal nature of diamond luminescence. These methods can in-

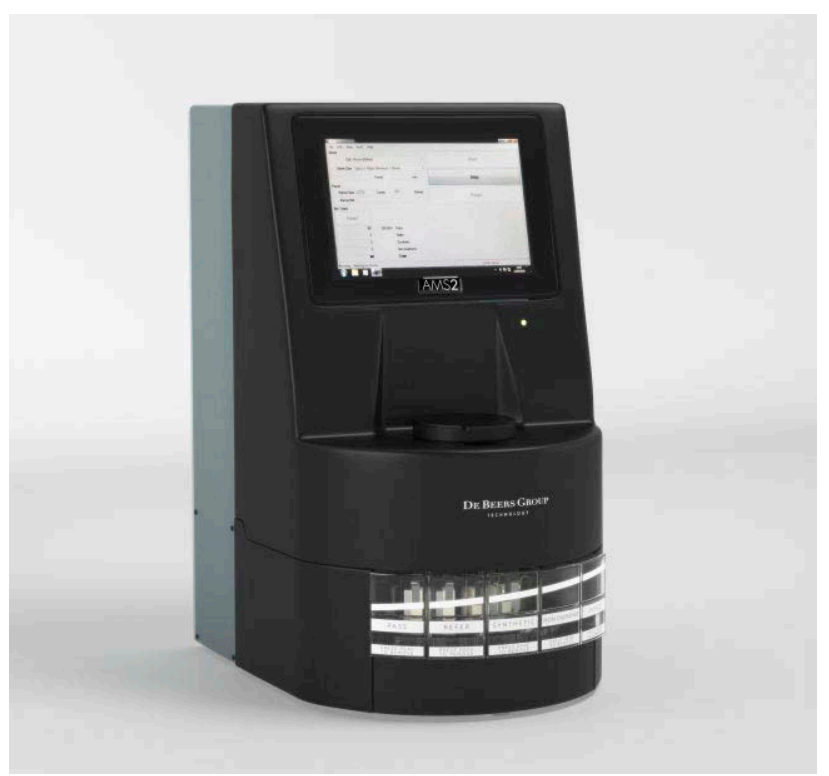

Figure 2. The De Beers AMS2, an instrument for automated screening of small colorless to near-colorless diamonds for potential synthetics and simulants at rates of up to 3,600 samples/hour. The size range is 0.003-0.20 ct for round brilliants and 0.01-0.20 ct for all other cuts. The instrument measures $31.5 \mathrm{~cm}$ wide $\times 40.0 \mathrm{~cm}$ deep $\times 55.0 \mathrm{~cm}$ high. Photo by Danny Bowler/De Beers Group Industry Services.

clude time-gated luminescence (Khong et al., 1994; Lindblom et al., 2003; Lipatov et al., 2007), singlephoton counting (Thomaz and Davies, 1978), and time-correlated single-photon counting (TCSPC) (Liaugaudas et al., 2009; Jones et al., 2020), to name a few. The method described in this article can be used to positively identify a natural type Ila or type Ia colorless diamond by accurately imaging a weak delayed luminescence having peak emission at $455 \mathrm{~nm}$ and a characteristic unquenched decay constant of $8.8 \mathrm{~ms}$. An example of a quenched (i.e., a decrease in the luminescence efficiency and a reduction of the measured decay time) luminescence decay, which has been observed in type IIa and type Ia natural diamonds, will be shown for comparison. The absence of the blue delayed luminescence, quenched or unquenched, would indicate that a sample may be type IIb natural, unusual type Ia natural, synthetic, or simulant and would require further testing to verify its origin. The De Beers Group screened many millions of individual colorless diamonds to verify this concept, and it is this principle that is used in both the De Beers SYNTHdetect jewelry screening instrument and the second version of the Automated Melee Screener (AMS2) (figures 1 and 2) (Martineau and McGuinness, 2018). 
More recently, this technique has identified a previously unknown green luminescence feature at 499 $\mathrm{nm}$ (Wassell et al., 2018) observable in lab-grown diamonds obtained from Gemesis Inc. (now Pure Grown Diamonds) and in silicon-containing samples sourced by Element Six that had undergone postgrowth annealing.

\section{MATERIALS AND METHODS}

A selection of colorless/near-colorless natural and lab-grown polished diamond samples were chosen for this study, the details of which are listed in table 1 . The samples are not an exhaustive set but were chosen to demonstrate the wide range of prompt and delayed luminescence characteristics that can be observed, and to provide a useful reference source for operators interpreting output from the SYNTHdetect instrument.

Microsecond time-gated imaging was carried out using a Teledyne Dalsa Genie Nano C2050 area-scan CMOS camera. Above-diamond band-gap excitation was provided by a Hamamatsu Photonics L7685 xenon flash lamp spectrally filtered to $190-227 \mathrm{~nm}$ output, with a temporal pulse width of $2.9 \mu$ s at full width half maximum. The camera and flash lamp were synchronized using the camera's internal timer, with the flash lamp signal offset by $11 \mu$ with respect to the timer to account for the difference in latency between the two devices. Images shown represent an average of 20 individual captures. These images are at $1.5 \times$ magnification as opposed to $0.25 \times$ in SYNTHdetect for the purposes of this publication.
TABLE 1. Diamond samples from this study.

\begin{tabular}{lll}
\hline Sample & Description & Shape and Cutting Style \\
\hline D1 & Type lla natural & Round brilliant \\
D2 & Green-fluorescing type la natural & Round brilliant \\
D3 & Unusual natural & Round brilliant \\
D4 & Weak type Ilb natural & Round brilliant \\
C1 & As-grown nitrogen-containing CVD & Round brilliant \\
C2 & CVD of Chinese origin & Round brilliant \\
C3 & Gemesis CVD synthetic & Modified square brilliant \\
C4 & Diamond Foundry CVD & Round brilliant \\
C5 & CVD of unknown origin & Round brilliant \\
S1 & Typical HPHT synthetic & Round brilliant \\
\hline
\end{tabular}

Room-temperature spectral data was collected by an Andor iStar DH320T-18U-E3 intensified CCD camera via a Horiba iHR-320 spectrometer, with excitation from the same source as the imaging setup. Synchronization with the flash lamp and camera was supplied by two externally generated pulsed signals offset in a similar way to the imaging setup. To record prompt luminescence, the intensifier delay was set to zero, with emission integrated over a 5 us gate. To record delayed luminescence, the intensifier delay was set to $100 \mu$ s or more, with emission integrated over a chosen gate. Here, 40 accumulations were averaged. A simplified diagram illustrating the principle of timegated luminescence is shown in figure 3 .

Selected room-temperature delayed luminescence decay data was collected by a Horiba Jobin Yvon IBH TBX-04 thermoelectrically cooled photomultiplier

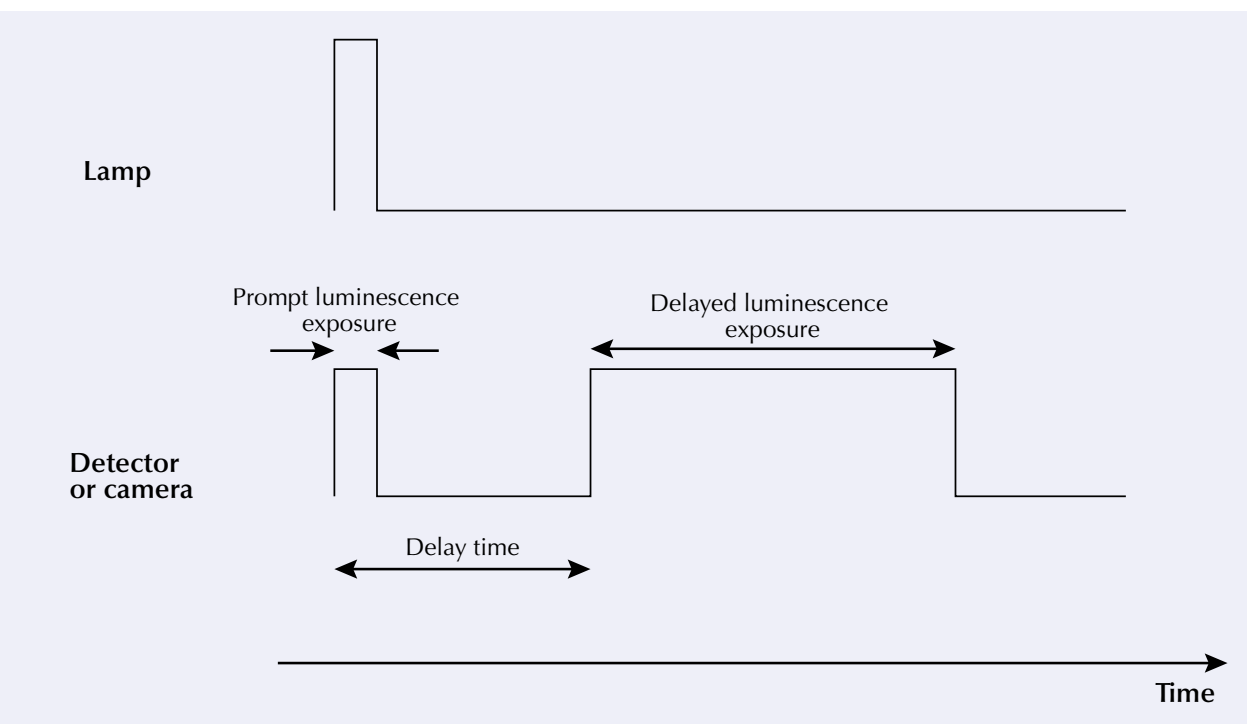

Figure 3. Simplified diagram of time-gated luminescence. To acquire a delayed spectrum or a delayed image, the detector or camera is activated after a delay, which ensures that the prompt luminescence has decayed almost to zero. Switching the detector or camera on and off is analogous to the opening and closing of a gate. To detect the prompt luminescence, and reject the delayed luminescence, the detector is turned on only when the lamp is on. 


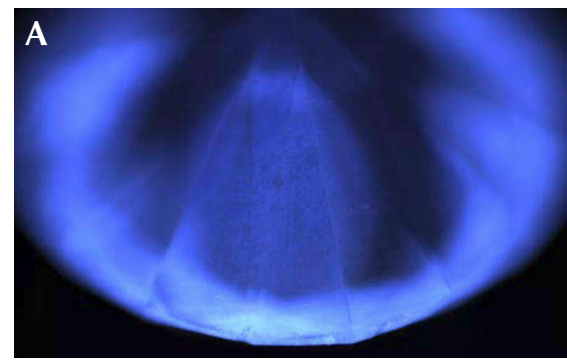

D

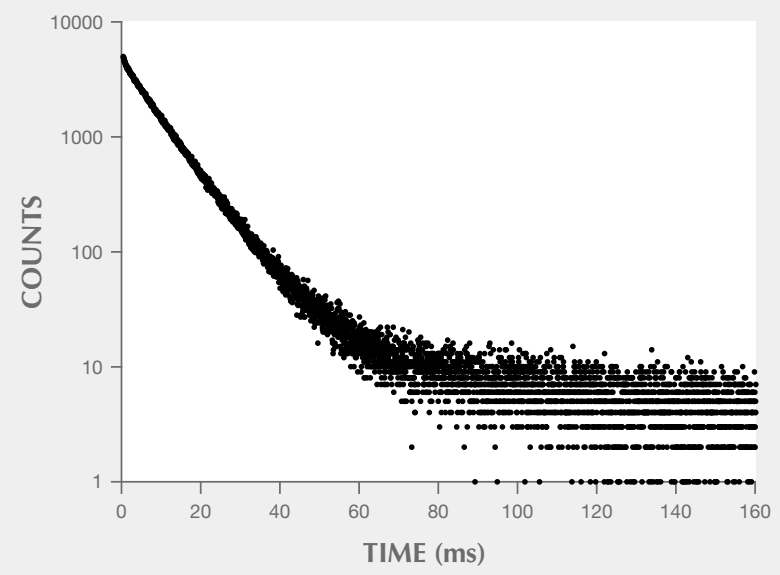

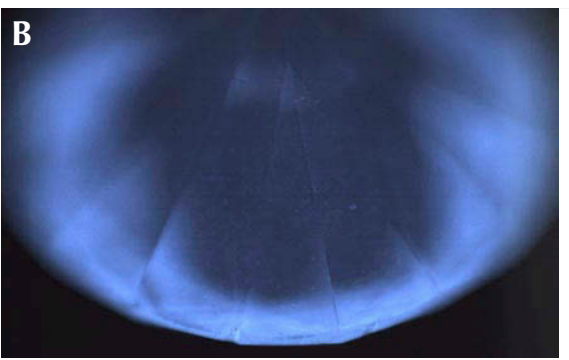

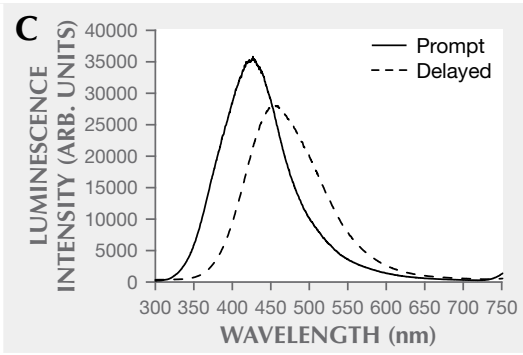

$\mathbf{E}$

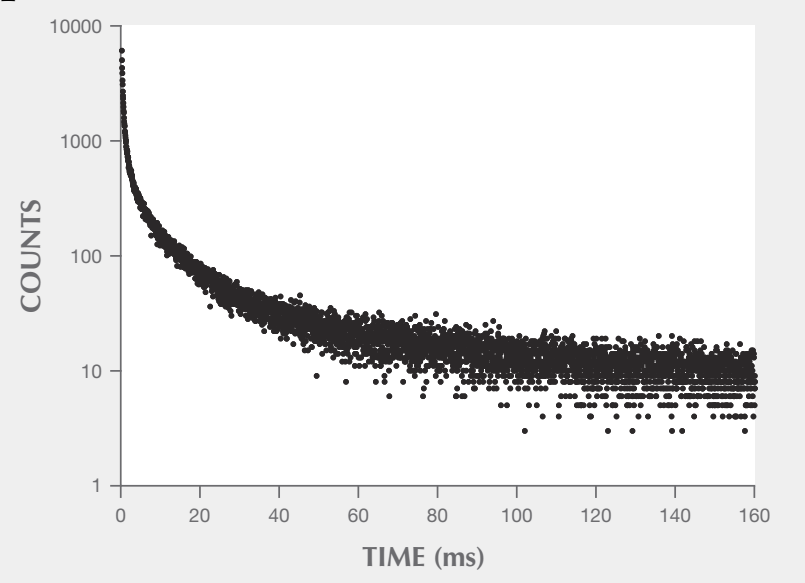

Figure 4. A: CMOS image of sample D1 showing the spatial distribution of the prompt blue dislocation-related luminescence signal exhibited by a typical type II a natural diamond. B: CMOS image showing the delayed luminescence signal exhibited. Recorded with a delay of $100 \mu \mathrm{s}$ after the rising edge of the UV pump pulse and integrated for $30 \mathrm{~ms}$. C: Prompt (peak at $435 \mathrm{~nm}$ ) and delayed (peak at $455 \mathrm{~nm}$ ) luminescence recorded for sample D1. Delayed luminescence was recorded with a delay of 100 us after the rising edge of the UV pump pulse and integrated for $30 \mathrm{~ms}$. D: Delayed luminescence decay profile of sample D1. In this example, the decay is unquenched and can be fitted to a simple monoexponential function with decay constant $8.8 \mathrm{~ms}$. Type Ia natural diamonds may also show unquenched decays. E: Example of a quenched decay seen in type IIa and type Ia natural diamonds. It would not be possible to distinguish this from an unquenched example using the imaging setup. At the time of this writing, it is not fully understood what processes would contribute to a quenching of this delayed luminescence.

module via a Horiba iHR320 spectrometer with excitation as above. Detector and flash lamp were triggered simultaneously, with channels recording both prompt and delayed luminescence in each time sweep of the multichannel analyzer. The time spacing of the channels was selected to suit the decay time being measured. After the data were accumulated, the channels recording prompt luminescence were rejected.

\section{RESULTS}

Natural Diamonds. The luminescence characteristics of a typical type IIa natural diamond are shown in the figure 4 set. The prompt luminescence (figure $4 \mathrm{~A}$ ) is dominated by blue luminescence from dislocations and is identical to that seen in the Diamond-
View instrument. The delayed luminescence (figure 4B) cannot be observed in DiamondView images due to the weak nature of the luminescence and the fact that it is masked by the prompt dislocation luminescence. It can be seen from the imaging that the blue delayed luminescence is not related to the blue dislocation prompt luminescence. The spectral data (figure 4C) and decay data (figure 4D) also show that it does not arise from N3 defect fluorescence. Figure 4E is an example of quenched blue delayed luminescence decay and is shown for comparison with the unquenched decay of sample D1. This blue delayed luminescence is the specific marker used by AMS2 and SYNTHdetect as a positive identifier of natural diamond, as it is not seen in synthetic diamonds. 

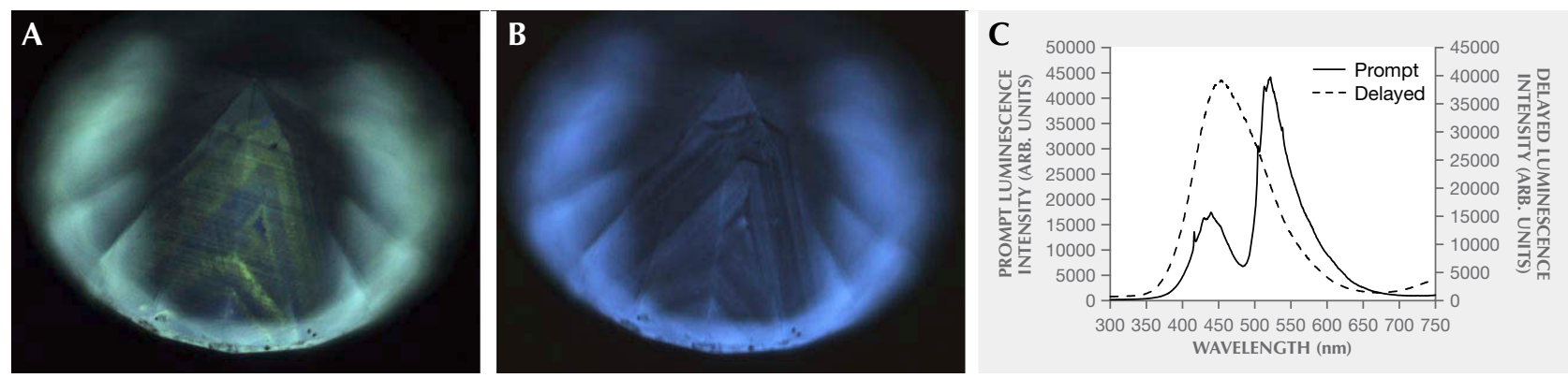

Figure 5. A: CMOS image of sample D2 showing the prompt green luminescence related to the H3 defect in type Ia natural diamond. Underlying prompt blue luminescence attributed to the N3 defect is also observed. B: CMOS image showing the blue delayed luminescence signal. In this case, structure is observed in the delayed luminescence signal, similar to the prompt luminescence signal. C: Prompt and delayed (peak at $455 \mathrm{~nm}$ ) luminescence recorded for sample D2 showing green prompt luminescence. Delayed luminescence recorded with a delay of $100 \mu \mathrm{s}$ after the rising edge of the UV pump pulse and integrated for $30 \mathrm{~ms}$.

This was confirmed in tests by De Beers Group on over 20 million individual colorless diamonds. In addition, the Diamond Producers Association ASSURE program found that less than $1 \%$ of natural diamonds do not exhibit this luminescence and would be referred for further testing (Dupuy and Phillips, 2019). At the time of writing, the origin of this luminescence is not fully understood.

The diamond in figure $5 \mathrm{~A}$ is a predominantly "green-fluorescing" example where the green prompt luminescence is attributed to the $\mathrm{H} 3$ defect and the blue to the $\mathrm{N} 3$ defect, as shown by the spectral data in figure 5C. The spectral data further show that the delayed luminescence is not related to N3 defect fluorescence, although the delayed luminescence image (figure 5B) shows that it is predominantly in the same spatial region of the stone as the N3 luminescence, which may suggest it is related to higher aggregated states of nitrogen such as B-centers.

Diamond D3 is a slightly more unusual natural diamond in that it exhibits blue prompt luminescence (figure 6A) and a blue/green delayed luminescence (figure 6B). The green delayed luminescence component is due to the $\mathrm{H} 3$ defect (figure 6D). This phenomenon has been reported previously and attributed to the population of triplet states within the defect (Pereira and Monterio, 1991). In this sample, the spectral data also show a small contribution of $\mathrm{H} 3$ in the prompt luminescence. By extending the delay from $100 \mu$ s to $2 \mathrm{~ms}$ (figure 6C), it can be seen that the delayed green luminescence from H3 decays much faster than the blue delayed luminescence used to identify natural diamond. Therefore, in examples such as these, increasing the camera delay time from the microsecond range to the millisecond range reduces the fast-decaying green components, allowing the underlying blue luminescence to be observed-a feature that is available to users of the SYNTHdetect instrument. Extensive testing by De Beers indicates that $~ 0.5 \%$ of natural diamonds would exhibit luminescence properties such as this.

The figure 7 set illustrates a typical example of a colorless weak type IIb natural diamond, sample D4. This would show turquoise-colored long-lived delayed luminescence in the DiamondView. In figure 7A, the underlying blue dislocation prompt luminescence can be clearly seen as the long-lived turquoise delayed luminescence shown in figure $7 \mathrm{~B}$ is gated out. This prompt luminescence would be nearly impossible to observe in the DiamondView due to the unsynchronized nature of the lamp and camera and the strong long-lived delayed luminescence, which would quickly mask the blue prompt luminescence. Indeed, a continual live feed of this prompt luminescence image is not possible in the DiamondView. Figure 7C illustrates the spectral profile, with the peak of the prompt luminescence at $425 \mathrm{~nm}$ and the peak of the delayed luminescence at $480 \mathrm{~nm}$ commonly seen in such diamonds. Due to the high resolution and higher magnification of these images, it is possible to identify this as natural diamond from the dislocation patterns in the prompt luminescence. However, in a lower-magnification system such as the SYNTHdetect, which is designed to look at an ensemble of diamonds in jewelry, the dislocation patterns may not be visible and such a diamond would need to be referred for further testing. This is due to the potential 

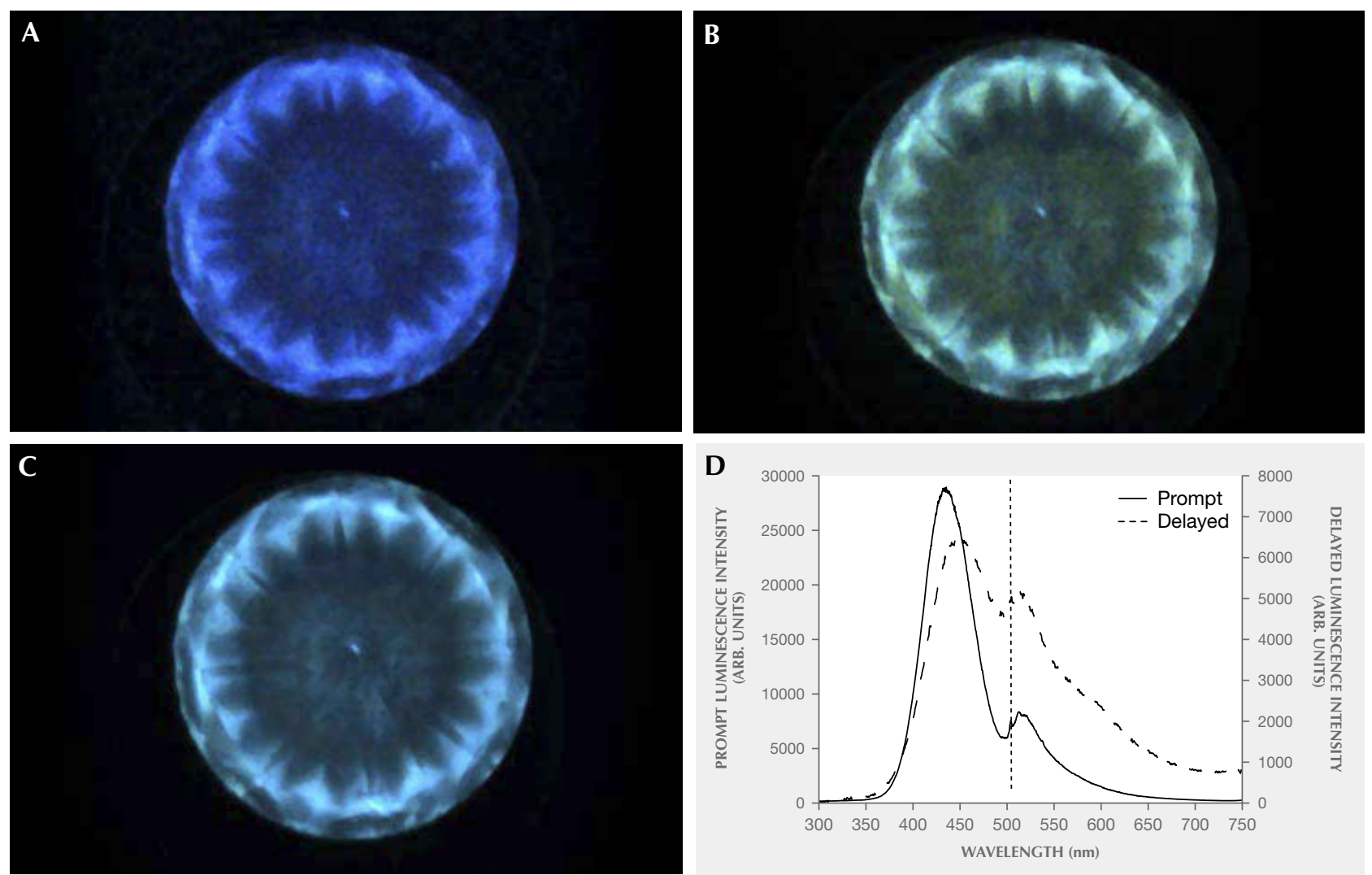

Figure 6. A: CMOS image of sample D3 showing the prompt luminescence from a slightly more unusual natural diamond. B: CMOS image of sample D3 showing a more unusual blue/green delayed luminescence signal in a natural diamond. Recorded with a delay of 100 us after the rising edge of the UV pump pulse and integrated for $30 \mathrm{~ms}$. C: CMOS image showing the delayed luminescence recorded with a longer delay of $2 \mathrm{~ms}$ after the rising edge of the UV pump pulse and integrated for $30 \mathrm{~ms}$. The green luminescence component has decayed. D: Prompt and delayed luminescence recorded for sample D3 showing both blue and green delayed luminescence. Delayed luminescence, recorded with a delay of $100 \mu \mathrm{s}$ after the rising edge of the UV pump pulse and integrated for $30 \mathrm{~ms}$, corresponds to figure 6B. The dotted line at $503 \mathrm{~nm}$ indicates the $\mathrm{H3}$ zero-phonon line common to prompt and delayed luminescence.

for certain synthetic types to produce prompt blue luminescence and delayed turquoise luminescence.
CVD Synthetic Diamonds. Sample C1 is a typical as-grown CVD synthetic diamond that displays or-

Figure 7. A: CMOS image of sample D4 showing the spatial distribution of the prompt blue dislocation-related luminescence signal exhibited by a typical type IIb natural diamond. B: CMOS image showing the delayed luminescence signal exhibited. Recorded with a delay of $100 \mu$ s after the rising edge of the UV pump pulse and integrated for $30 \mathrm{~ms}$. C: Prompt and delayed (peak at $480 \mathrm{~nm}$ ) luminescence recorded for sample D4. Delayed luminescence recorded with a delay of 100 us after the rising edge of the UV pump pulse and integrated for $30 \mathrm{~ms}$.
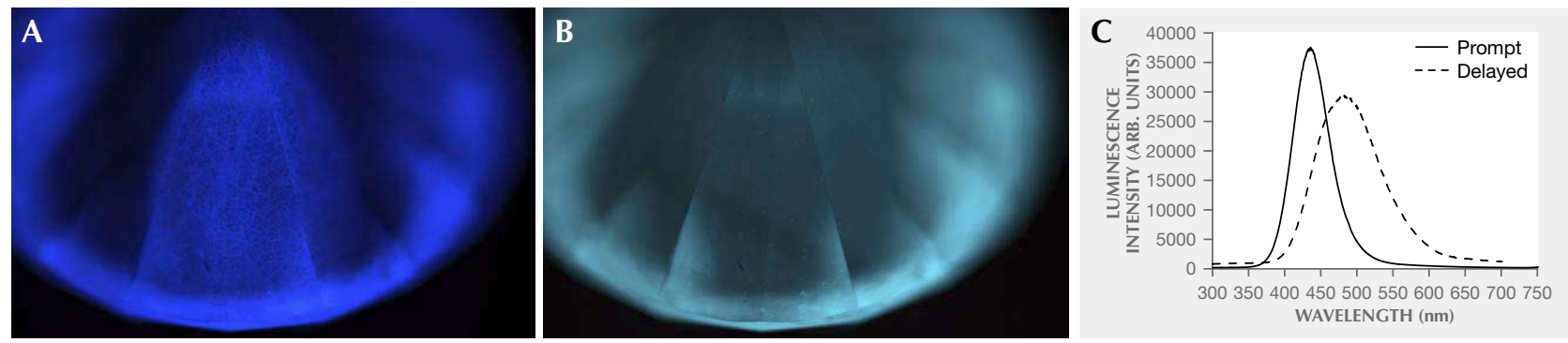

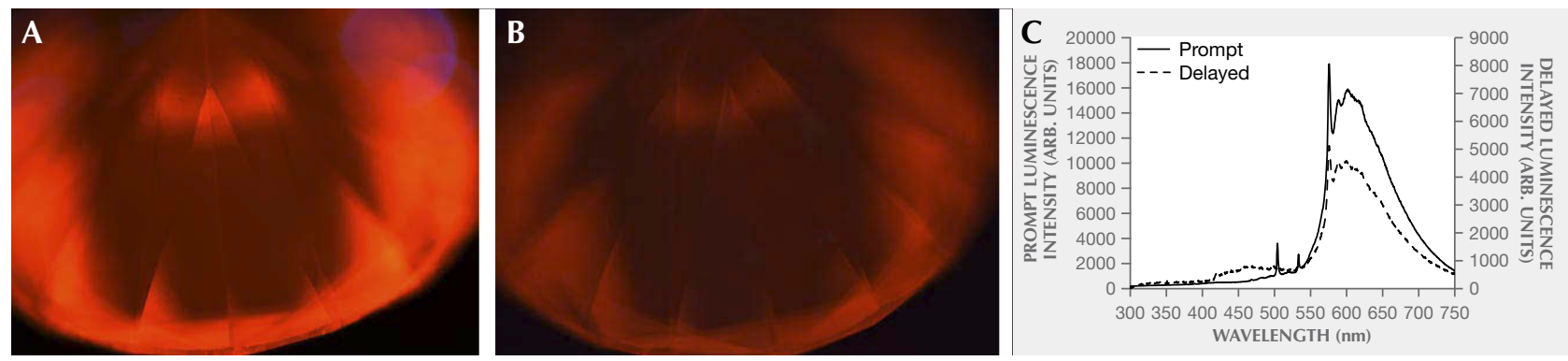

Figure 8. A: CMOS image of sample C1 showing the prompt luminescence from a typical as-grown CVD synthetic diamond. B: CMOS image showing the delayed luminescence signal exhibited. Recorded with a delay of 100 us after the rising edge of the UV pump pulse and integrated for $60 \mathrm{~ms}$. C: Prompt and delayed luminescence recorded for sample C1. Delayed luminescence recorded with a delay of 100 us after the rising edge of the UV pump pulse and integrated for $60 \mathrm{~ms}$.

ange prompt luminescence (figure 8A), primarily from the $\mathrm{NV}^{0}$ defect (figure $8 \mathrm{C}$ ). An interesting effect noticed in this type of diamond is the delayed orange luminescence (figure 8B). Spectral analysis shows that this also originates from the $\mathrm{NV}^{0}$ defect. It can be concluded that the $\mathrm{NV}^{0}$ defect can experience emission from spin-forbidden transitions in a way similar to the $\mathrm{H} 3$ defect (Pereira and Monterio, 1991), although this discussion is beyond the scope of this article.

Diamond C2 is a commercially available CVD synthetic made in China. Figure 9A shows blue dislocation luminescence with discrete narrow bands and a weak general underlying red luminescence. Spectral data in figure 9C shows that the red prompt luminescence is predominantly from the $\mathrm{NV}^{0}$ defect. The delayed green luminescence in this example (figure 9B) is due to the H3 defect. Delayed luminescence from the $\mathrm{H} 3$ defect has been discussed for the natural diamond D3 (again, see figure 6). It is also a good indication that this synthetic diamond has undergone post-growth annealing, as this delayed luminescence has been previously observed in such samples that have undergone annealing at around $1700^{\circ} \mathrm{C}$ (Wassell et al., 2018).

Sample C3 is a commercially available CVD synthetic diamond from Gemesis that has been reported previously (Wassell et al., 2018). At first glance, the delayed luminescence CMOS image (figure 10B) appears very similar to that of sample $\mathrm{C} 2$ in figure 9. However, the spectral data highlight that this luminescence, although similar in color, originates from an entirely different defect, with a zero-phonon line at $499 \mathrm{~nm}$ (figure 10C). This defect has been shown to be generated by post-growth annealing at around $1700^{\circ} \mathrm{C}$. This sample is of interest in diamond verification, as it exhibits blue prompt luminescence from dislocation patterns in local regions (figure 10A) and could be incorrectly identified as a type IIa natural by a low-magnification system or an instru-

Figure 9. A: CMOS image of sample C2 showing the prompt luminescence from a commercially available CVD synthetic of Chinese origin. B: CMOS image showing the delayed luminescence signal exhibited. Recorded with a delay of $100 \mu \mathrm{s}$ after the rising edge of the UV pump pulse and integrated for $60 \mathrm{~ms}$. C: Prompt and delayed luminescence recorded for sample C2. Delayed luminescence recorded with a delay of $100 \mu$ s after the rising edge of the UV pump pulse and integrated for $60 \mathrm{~ms}$.
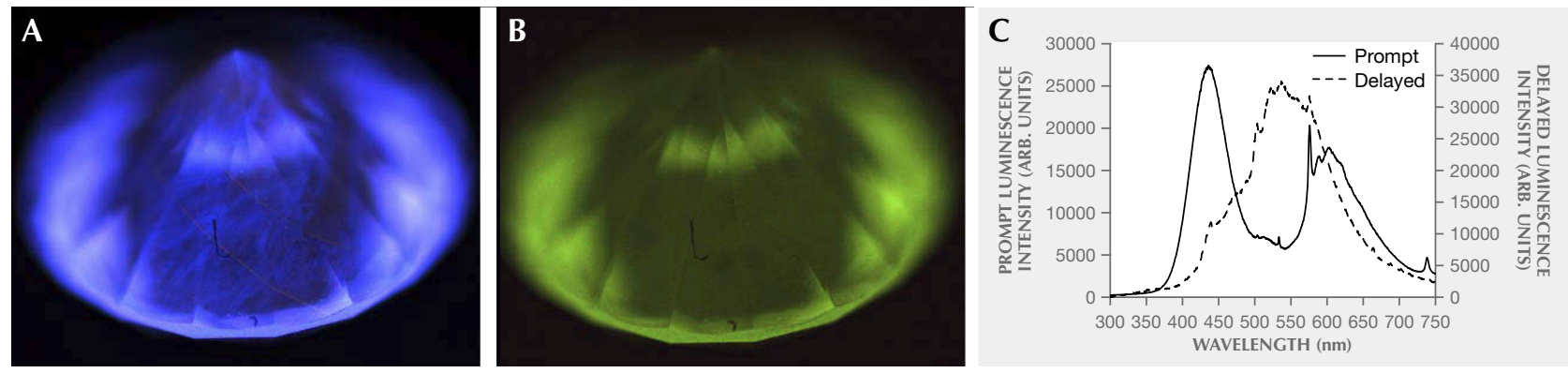

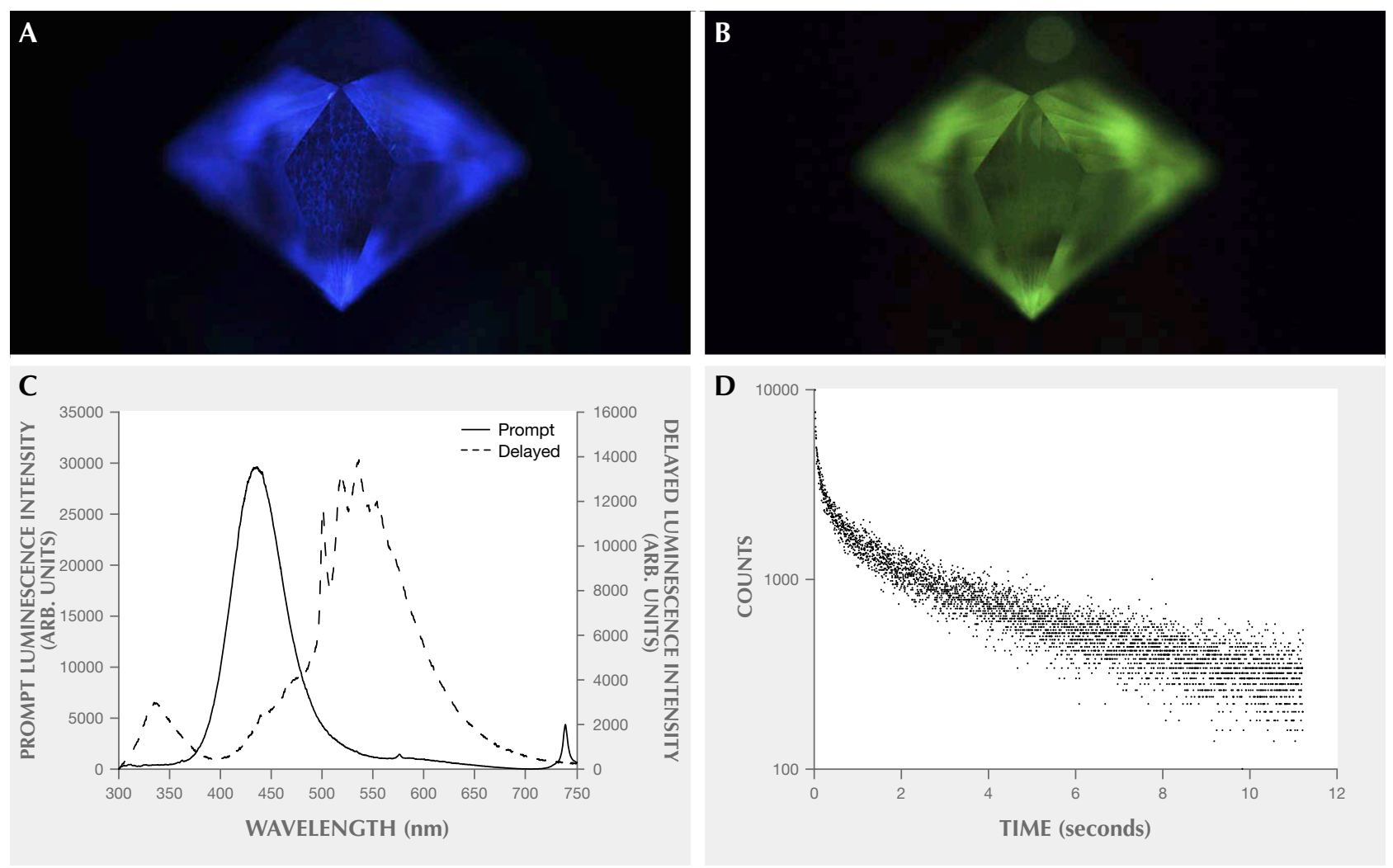

Figure 10. A: CMOS image of sample C3 showing the spatial distribution of the prompt blue luminescence signal for a commercially available Gemesis CVD synthetic. B: CMOS images of the delayed 499 nm luminescence signal, with a delay of $100 \mu \mathrm{s}$ after the rising edge of the UV pump pulse and integrated for $60 \mathrm{~ms}$. C: Prompt and delayed (499 nm ZPL) luminescence recorded for sample C3. Delayed luminescence recorded with a delay of $100 \mu \mathrm{s}$ after the rising edge of the UV pump pulse and integrated for $60 \mathrm{~ms}$. D: Delayed luminescence decay profile of a blue-fluorescing Si-containing CVD synthetic. In this example, the decay is complex and non-exponential. An average decay time of $\sim 1 \mathrm{sec}$ is observed.

ment with a low-resolution camera where blue fluorescence is used as a natural identifier. Figure 10D shows the delayed luminescence decay profile for the $499 \mathrm{~nm}$ feature. It should not be assumed, however, that this observed decay is the decay profile for the $499 \mathrm{~nm}$ feature in general. Rather, it is the decay profile of the $499 \mathrm{~nm}$ feature in this sample. It was also suggested by Wassell et al. (2018) that prompt luminescence of $499 \mathrm{~nm}$ could not be ruled out due to swamping by blue prompt luminescence.

A commercially available CVD synthetic diamond from Diamond Foundry (sample C4) shows both a green prompt and green delayed luminescence (figures 11A and 11B). Again, this could be attributed to the $\mathrm{H} 3$ defect, but spectral data (figure $11 C$ ) show that this is a result of the $499 \mathrm{~nm}$ feature in both prompt and delayed luminescence.

Sample C5 is a commercially available CVD diamond of unknown origin. Figure 12A shows growth bands roughly perpendicular to the growth direction in the prompt luminescence, consistent with CVD synthesis. The spectral data presented in figure 12C show that this luminescence is predominantly from the $499 \mathrm{~nm}$ feature with components at $575 \mathrm{~nm}$ (the $\mathrm{NV}^{0}$ defect) and at $415 \mathrm{~nm}$ (the N3 defect), although this level of imaging does not show these defects in specific localized areas. Interestingly, the delayed luminescence seen in figure $12 \mathrm{~B}$ does not show as prominent growth band patterns, suggesting the delayed turquoise luminescence does not form these patterns in the same way as the prompt luminescence. The spectral data show that the delayed luminescence is dominated by the broad turquoise feature with only a small level of the $499 \mathrm{~nm}$ feature visible. This sample also indicates that the $499 \mathrm{~nm}$ feature can experience both prompt and delayed emission.

HPHT Synthetic Diamond. Sample S1 is an example of a typical commercially sourced HPHT syn- 

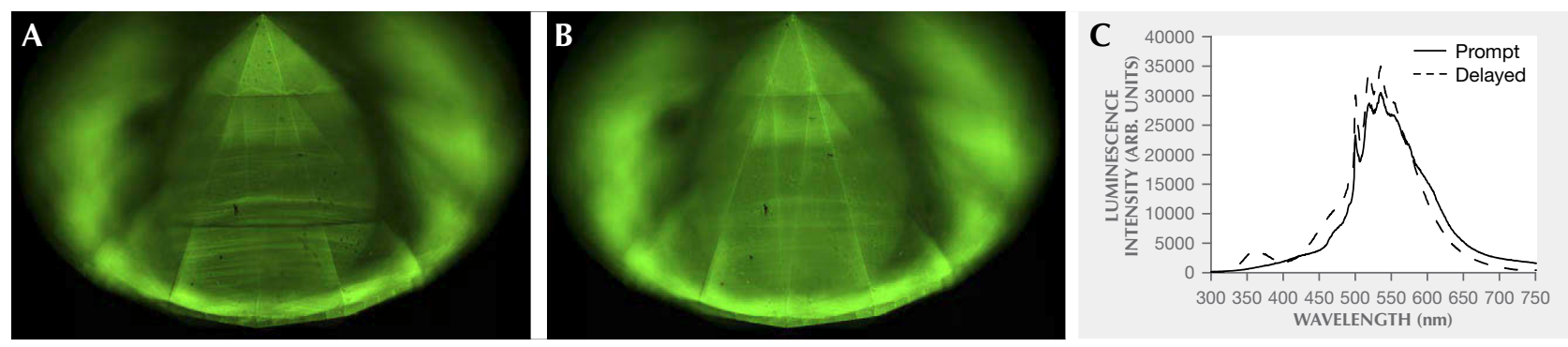

Figure 11. A: CMOS image of sample C4 showing prompt green luminescence from the 499 nm feature for a commercially available Diamond Foundry CVD synthetic. B: CMOS image of the delayed 499 nm luminescence signal, with a delay of 100 us after the rising edge of the UV pump pulse and integrated for 60 ms. C: Prompt and delayed luminescence recorded for sample C4. Delayed luminescence recorded with a delay of $100 \mu \mathrm{s}$ after the rising edge of the UV pump pulse and integrated for $60 \mathrm{~ms}$.

thetic diamond. The delayed luminescence (figure $13 \mathrm{~B}$ ) is identical to the strong long-lived luminescence that would be observed in the DiamondView. This long-lived luminescence is understood and has been reported as being interpretable in terms of a donor-acceptor recombination model (Watanabe et al., 1997). Of interest here is the prompt luminescence (figure 13A), which is a weak green/red and would normally be obscured by the long-lived luminescence in the DiamondView. This luminescence could be due to the presence of metal ions in the solvent catalyst. However, the prompt luminescence spectrum shown in figure 13C, along with the delayed luminescence, is broad and featureless at room temperature and gives little in the way of information.

This weak green prompt luminescence and strong long-lived turquoise luminescence are typical of many HPHT synthetics. They can be used as an iden- tifying feature, as this combination of luminescence features have not been seen in natural diamond.

\section{CONCLUDING COMMENTS}

Diamond screening is becoming increasingly challenging, and organizations other than gemological laboratories, such as independent jewelers, auction houses, and pawnbrokers, are charged with this important task. Not only are more sophisticated techniques required to assist with this challenge, but the equipment must not be so complex to use and difficult to understand that diamond screening becomes an overly time-consuming and inefficient task. In this article, a selection of natural and lab-grown polished colorless diamonds has been studied using deep-UV excitation where the recording of the luminescence is synchronized with the excitation source, allowing a time-gated measurement. This technique provides a useful additional level of verification when screening

Figure 12. A: CMOS image of sample C5 showing the spatial distribution and growth bands perpendicular to the growth direction in the prompt green luminescence signal exhibited by a commercially available CVD synthetic diamond. B: CMOS image showing the delayed turquoise-colored luminescence signal exhibited. The growth patterns in the prompt luminescence are less prominent here. C: Prompt and delayed luminescence recorded for sample C5. Delayed luminescence recorded with a delay of $100 \mu \mathrm{s}$ after the rising edge of the UV pump pulse and integrated for $60 \mathrm{~ms}$. The inset zooms in on the region of the $499 \mathrm{~nm} Z \mathrm{PL}$.
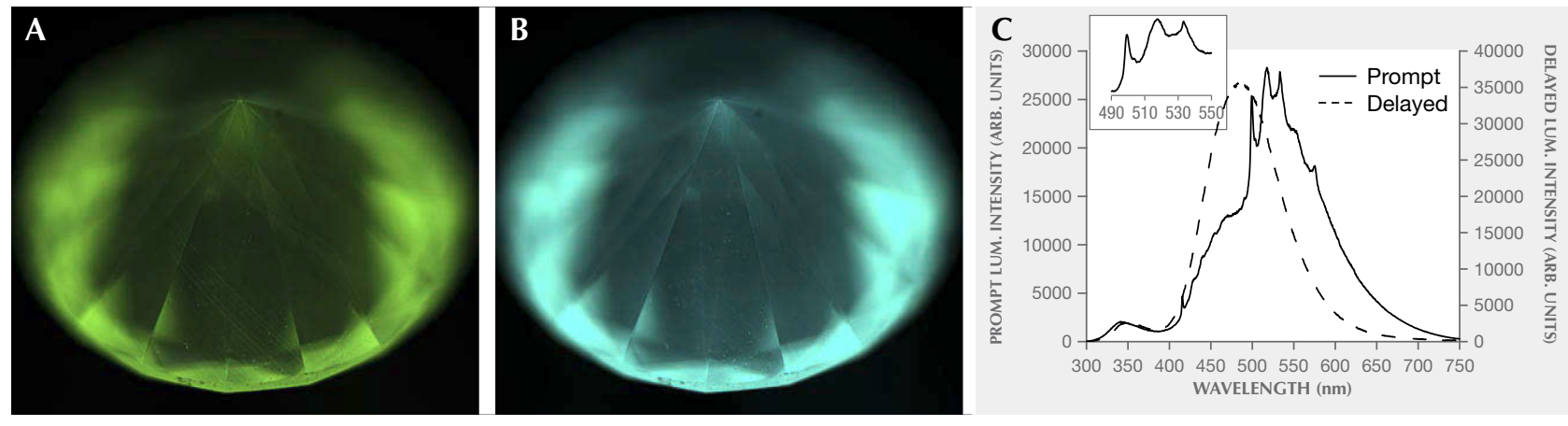

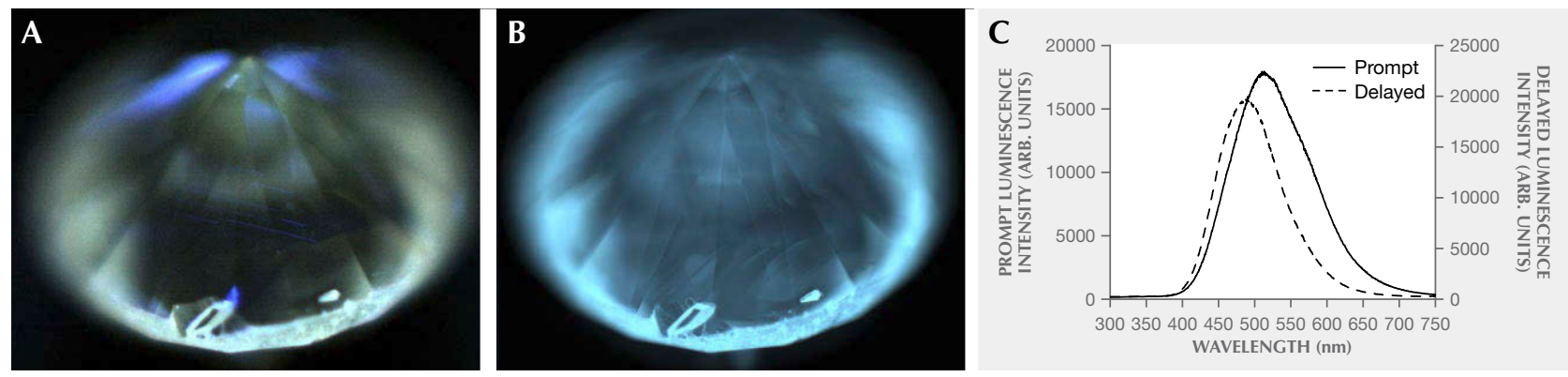

Figure 13. A: CMOS image of sample S1 showing a prompt green/red luminescence from a typical colorless HPHT synthetic diamond. B: CMOS image of sample S1 showing the delayed luminescence from a typical colorless HPHT synthetic-normally attributed to the presence of boron. C: Prompt (peak $\sim 513 \mathrm{~nm}$ ) and delayed (peak 480 $\mathrm{nm}$ ) luminescence recorded for sample S1. Delayed luminescence recorded with a delay of $100 \mu \mathrm{s}$ after the rising edge of the UV pump pulse and integrated for $10 \mathrm{~ms}$.

for challenging synthetics such as blue-fluorescing CVD synthetics. Although the examples contained in this article are not an exhaustive list, they should serve as a useful reference guide and knowledge base for all SYNTHdetect users when studying individual samples or screening challenging examples.
ABOUT THE AUTHORS

Dr. McGuinness is a senior scientist, and Dr. Lanigan is a research scientist, at De Beers Group Technology, Maidenhead, Berkshire, United Kingdom. Ms. Wassell is a doctoral student, and Dr. Lynch is associate dean of research, at the School of Physics \& Astronomy, Cardiff University, Cardiff, United Kingdom.

\section{ACKNOWLEDGMENTS}

The authors would like to thank Mr. Hugh Leach at De Beers Group Technology for mechanical engineering and instrument development effort, and to the team at Element Six whose samples were used extensively in the development of this technique.

\section{REFERENCES}

Dupuy H., Phillips J.C. (2019) Selecting a diamond verification instrument based on the results of the Assure program: An initial analysis. Journal of Gemmology, Vol. 36, pp. 606-619.

Jones D.C., Kumar S., Lanigan P.M.P., McGuinness C.D., Dale M.W., Twitchen D.J., Fisher D., Martineau P.M., Neil M.A.A., Dunsby C., French P.M.W. (2020) Multidimensional luminescence microscope for imaging defect colour centres in diamond. Methods and Applications in Fluorescence, Vol. 8, No. 1, 014004, http://dx.doi.org/10.1088/2050-6120/ab4eac

Khong Y.L., Collins A.T., Allers L. (1994) Luminescence decay time studies and time-resolved cathodoluminescence spectroscopy of CVD diamond. Diamond and Related Materials, Vol. 3, No. 7, pp. 1023-1027, http://dx.doi.org/10.1016/09259635(94)90112-0

Liaugaudas G., Collins A.T., Suhling K., Davies G., Heintzmann R. (2009) Luminescence-lifetime mapping in diamond. Journal of Physics: Condensed Matter, Vol. 21, No. 36, 364210, http://dx.doi.org/10.1088/0953-8984/21/36/364210

Lindblom J., Hölsä J., Papunen H., Häkkänen H., Mutanen J. (2003) Differentiation of natural and synthetic gem-quality diamonds by luminescence properties. Optical Materials, Vol. 24, No. 1-2, pp. 243-251, http://dx.doi.org/10.1016/S0925-3467/03/00130-7

Lipatov E.I., Lisitsyn V.M., Oleshko V.I., Tarasenko V.F. (2007) Spectral and kinetic characteristics of the pulsed cathodoluminescence of a natural IIa-type diamond. Russian Physics Journal, Vol. 50, No. 1, pp. 52-57, http://dx.doi.org/10.1007/s11182-0070005-8

Martineau P.M. (2017) The 68th Diamond Conference. University of Warwick, T2.1, July 10-13.

Martineau P.M., McGuinness C.D. (2018) De Beers consumer con- fidence technical research and diamond verification instruments. Diamonds - Source to Use Conference 2018, Southern African Institute of Mining and Metallurgy, Johannesburg, June $11-13$, pp. 35-44.

Martineau P.M., Lawson S.C., Taylor A.J., Quinn S.J., Evans D.J.F., Crowder M.J. (2004) Identification of synthetic diamond grown using chemical vapor deposition (CVD). Ge G, Vol. 40, No. 1, pp. 2-25, http://dx.doi.org/10.5741/GEMS.40.1.2

Pereira E., Monterio T. (1991) Delayed luminescence of the $\mathrm{H}_{3}$ centre in diamond. Journal of Luminescence, Vol. 48-49, pp. 814818, http://dx.doi.org/10.1016/0022-2313(91)90248-T

Smith J.G.C., McGuinness C.D., Fisher D. (2017) Luminescence measurements in diamond. Patent Application WO/2017/001835 Spear P.M., Welbourn C.M. (1994) Distinguishing natural from synthetic diamond. Patent Application WO/83/00389A1

Thomaz M.F., Davies G. (1978) The decay time of N3 luminescence in natural diamond. Proceedings of the Royal Society A, Vol. 362, No. 1710, pp. 405-419, http://dx.doi.org/10.1098/rspa.1978.0141

Wassell A.M., McGuinness C.D., Hodges C., Lanigan P.M.P. Fisher D., Martineau P.M., Newton M.E., Lynch S.A. (2018) Anomalous green luminescent properties in CVD synthetic diamonds. Physica Status Solidi A, Vol. 215, No. 22, 1800292, http://dx.doi.org/10.1002/pssa.201800292

Watanabe K., Lawson S.C., Isoya J., Kanda H., Sato Y. (1997) Phosphorescence in high-pressure synthetic diamond. Diamond and Related Materials, Vol. 6, No. 1, pp. 99-106, http://dx.doi.org/10.1016/S0925-9635/96)00764-9

Welbourn C.M, Cooper M., Spear P.M. (1996) De Beers natural versus synthetic diamond verification instruments. $G e G$, Vol. 32, No. 3, pp. 156-169, http://dx.doi.org/10.5741/GEMS.32.3.156 